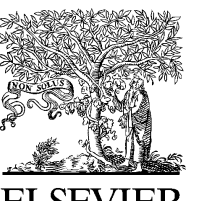

\title{
Trigonometric function used to formulate a multi-nodal finite tubular element
}

\author{
E.M.M. Fonseca ${ }^{\text {a,* }}$, F.J.M.Q. de Melo ${ }^{\text {b }}$, C.A.M. Oliveira ${ }^{\mathrm{c}}$ \\ a Department of Applied Mechanics, Campus de Sta. Apolónia Ap. 1134, Polytechnic Institute of Bragança, \\ 5301-857 Bragança, Portugal \\ ${ }^{\mathrm{b}}$ Department of Mechanical Engineering, University of Aveiro, 3810-193 Aveiro, Portugal \\ ${ }^{\mathrm{c}}$ Department of Mechanical Engineering, Faculty of Engineering of University of Porto, 4200-465 Porto, Portugal
}

Abstract

It is presented an alternative formulation to solve the problem of the deformation analysis for tubular element under pinching loads. The solution is based on a new displacement field defined from a total set of trigonometric functions. The solution is developed in a multi-nodal finite tubular ring element with a total of eight degrees of freedom per section considered. The purpose of this paper is to provide an easy alternative formulation when compared with a complex finite shell element or beam element analysis for the same application. Several case studies presented have been compared and discussed with numerical analyses results reported by other authors and the results obtained with a shell element from a Cos$\operatorname{mos} / \mathbf{M}^{\circledR}$ programme.

(c) 2006 Published by Elsevier Ltd.

Keywords: Multi-nodal; Finite tubular ring element; Pinching loads; Trigonometric functions

\section{Introduction}

Structural design methodologies for pipelines systems start to be used, especially when it is expected that enhancements in the design process will lead to more cost effective and durable designs. These piping elements exhibit complex deformations fields given their toroidal geometry and the multiplicity of the configuration of external loads. Mathematical and experimental models which permit to verify the flexibility of pipe systems were published for a long time by some researchers working in this scope: (von Kárman, 1911; Vigness, 1943; Cheng and Thailer, 1970) and more recently (Thomson, 1980; Wilczek, 1984; Bathe and Almeida, 1982). News models used for stress and displacements fields determination, under mechanical or thermal loads, were recently formulated using numerical techniques with new finite elements by Melo and Castro (1992), Fonseca et al. (2002, 2005). Studies on circular cylindrical shells under concentrated forces have been presented by many researchers using curved beam, shell and solid elements (Kouhia and Stenberg, 2000; Gob-

\footnotetext{
* Corresponding author. Tel.: +351 273303155; fax: +351 273313051.

E-mail addresses: efonseca@ipb.pt (E.M.M. Fonseca), fqm@mec.ua.pt (F.J.M.Q. de Melo), cmoliv@fe.up.pt (C.A.M. Oliveira).
} 
etti and Nascimbene, 2001). Shell element formulations based on the so-called degenerate models introduced by Ahmad et al. (1970) have been used due their ability in moderately thick and thin plate or shell elements A long report about the development of finite shell or plate elements with emphasis on innovations was presented by MacNeal (1998). News formulations of solid element were presented for the linear and nonlinear analysis of thin-walled structures by Kim et al. (2004). The particular case of the formulation of cylindrical shells using ring elements has a straightforward contribution from Oñate (1992), where this author used a combined formulation for the displacement field dealing with fourier series along the circumference and algebraic shape functions along the axial direction. Analysis of a circular ring is reasonable for the structural design of most buried pipes when they can withstand the three most basic loads: internal pressure, transportation/installation and external pressure (Watkins and Anderson, 2000). The ring deflection is a potential performance limit for transportation/installation of pipes. The aim of this study is to develop an alternative numerical method and present an experimental work for pipes analysis when used in underground sewerage without internal pressure or when submitted to mechanical loads imposed during transportation or an installation. Satisfactory results for circular rings can be obtained by several different methods. This work presents an alternative formulation based on one finite element with two nodal sections used to simulate pinching loads in the referred situations and analyse the structural mechanics of pipes in linear bending conditions.

\section{Finite element formulation: assumptions and deformation model}

The geometric parameters considered for this element definition are: the length of the pipe $s$, the wall thickness $t$ and the mean section radius of the pipe $r$. Fig. 1 shows the essential parameters and the degrees of freedom used to define the finite tubular ring element.

The basic kinematics assumptions refer to the deformation of a thin shell as used in a small-defection analysis. The assumptions in the problem formulation of in-plane bending are: the shell is thin, this meaning that the normal to the shell surface does not distort and the transverse section is inextensible, not including pressure effects. The strain analysis and curvature geometry field of a non-symmetrically deformed cylindrical shell may be founded on the behaviour of plates and shells of revolution. Kinematics expressions relating the midsurface strains are expressed by (1-3) and the curvatures and twist by (4-5).

$$
\begin{gathered}
\varepsilon_{s s}=\frac{\partial u}{\partial s}, \quad \varepsilon_{\theta \theta}=\frac{1}{r}\left(\frac{\partial v}{\partial \theta}+w\right) \quad \text { and } \quad m a_{s \theta}=\frac{1}{r} \frac{\partial u}{\partial \theta}+\frac{\partial v}{\partial s} \\
\chi_{\theta \theta}=\frac{1}{r^{2}}\left(-\frac{\partial v}{\partial \theta}+\frac{\partial^{2} w}{\partial \theta^{2}}\right) \quad \text { and } \quad \chi_{s \theta}=\frac{2}{r}\left(-\frac{\partial v}{\partial s}+\frac{\partial^{2} w}{\partial s \partial \theta}\right)
\end{gathered}
$$

where $\varepsilon_{\mathrm{ss}}$ is the longitudinal membrane strain, $\varepsilon_{\theta \theta}$ is the meridional curvature from ovalization, $\gamma_{\mathrm{s} \theta}$ the shear strain, $\chi_{\theta \theta}$ is the meridional curvature variation from ovalization and $\chi_{\mathrm{s} \theta}$ the twist variation.

A simplified theory is useful under certain conditions and is applicable to a variety of shells forms. However, we shall deal only with the inextensional deformation of circular cylindrical shells. This theory is often
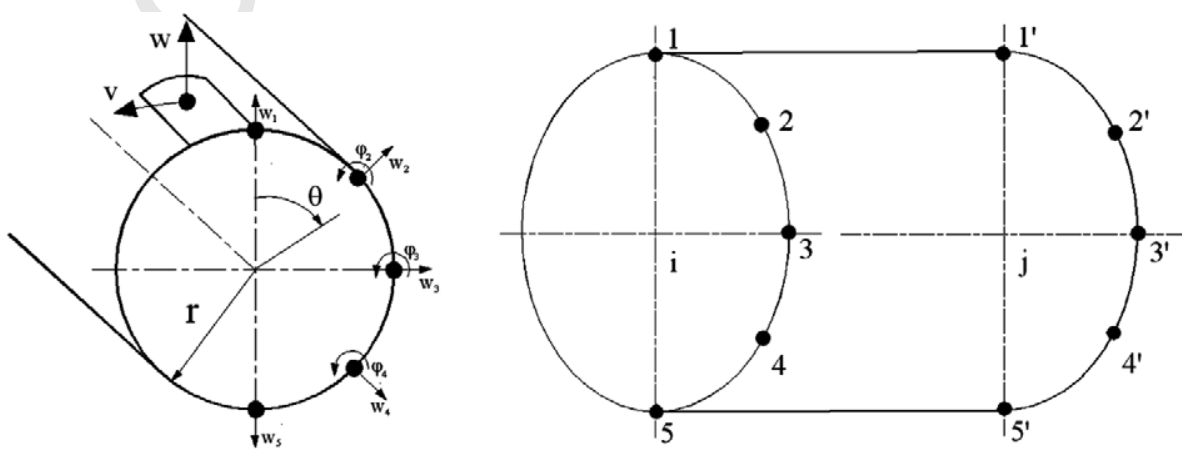

Fig. 1. Geometry and degrees of freedom of the finite tubular ring element. 
preferred when shell structures resist loading principally through bending action. Such cases include a cylinder subjected to loads without axial symmetry and confined to a small circumferential portion and there is considerable bending caused by changes in curvature, but no stretching of midsurface length. Deformations of these types are thus described as inextensional referred by Ugural (1981). In this shell theory, the midsurface in-plane strain components given by Eqs. (1-3) are taken to be zero. We realise from Eq. (1) that u depends on $\theta$ and Eq. (2) leads to:

$$
w=-\partial v / \partial \theta
$$

\section{The displacement field for the finite tubular ring element}

The displacement filed proposed characterises a uniform ovalization in the tube and a variation along the shell length. To obtain the shape functions a displacement field has been considered in the normal radial direction. As represented in Fig. 1 the element presents five nodes under symmetric conditions make possible studies in-plane bending. So, only a half of a ring section is needed to consider when trigonometric even functions are used. The initial radial displacement used to define the ovalization effect must be calculated using the trigonometric polynomial approximation. A formulation based on trigonometric functions is used and eight parameters are necessary to define the transversal displacement field approximated by:

$$
w(s, \theta)=\sum_{i=2}^{9} a_{i-1} i \cos i \theta+S \sum_{i=2}^{9} b_{i-1} i \sin i \theta
$$

Using simple differential equation from beam bending theory the transverse displacement can be calculated:

$$
v(s, \theta)=-\sum_{i=2}^{9} a_{i-1} \sin i \theta-S \sum_{i=2}^{9} b_{i-1} \cos i \theta
$$

The rotation field is considered using the derivative function of the transverse displacement field:

$$
\varphi(s, \theta)=\frac{1}{r} \frac{\partial w}{\partial \theta}=-\frac{1}{r} \sum_{i=2}^{9} a_{i-1} i^{2} \sin i \theta-\frac{S}{r} \sum_{i=2}^{9} b_{i-1} i^{2} \cos i \theta
$$

The unknown parameters $a_{i}$ are determined by imposing boundary conditions according to the ring element section $i$ and $j$ considered, resulting a system of equations to be solved. The degrees of freedom considered in the proposed element are: node 1 and 5 are the transversal displacement and their derivatives vanish, for nodes 2 at 4 have a radial displacement and one derivative function. A system with 16 equations is solved for $i$ and $j$ section of the ring element and shapes functions are obtained:

$$
\{\delta\}=\left[B^{\prime}\right]\left\{a_{i}\right\}
$$

with $\quad\{\delta\}^{\mathrm{T}}=\left\{\begin{array}{llllllllllllllll}W_{1 i} & W_{2 i} & \varphi_{2 x} & W_{3 i} & \varphi_{3 i} & W_{4 i} & \varphi_{4 i} & W_{5 i} & W_{1 j} & W_{2 j} & \varphi_{2 j} & W_{3 j} & \varphi_{3 j} & W_{4 j} & \varphi_{4 j} & W_{5 j}\end{array}\right\}^{\mathrm{T}}$ which represents the global displacement field for transversal and rotation degrees of freedom in the semi nodal ring and $\left[B^{\prime}\right]$ is constant matrix that results from the imposed boundary conditions. For node 1 the transversal displacement is equal to one and all others equal to zero. The first shape function appears and is called $N_{1 i}$. With imposed Eq. (6) we determine the shape function $B N_{1 i}$. The same has been used for all others nodes. The unknown constants are determined inverting the system equation (10). The first shape function is represented by Eq. (11). With these conditions, a new shape functions are determined and the generic local displacements field for in-plane finite element formulation are given by Eqs. (12) and (13).

$$
\begin{aligned}
N_{1 i}= & {\left[\frac{3}{16} \cos (2 \theta)+\frac{5}{32} \cos (3 \theta)+\frac{1}{8} \cos (4 \theta)+\frac{3}{32} \cos (5 \theta)+\frac{1}{16} \cos (6 \theta)+\frac{9}{64} \cos (7 \theta)+\frac{1}{8} \cos (8 \theta)\right.} \\
& \left.+\frac{7}{64} \cos (9 \theta)\right]-s\left[\frac{3}{16} \frac{\cos (2 \theta)}{L}+\frac{5}{32} \frac{\cos (3 \theta)}{L}+\frac{1}{8} \frac{\cos (4 \theta)}{L}+\frac{3}{32} \frac{\cos (5 \theta)}{L}+\frac{1}{16} \frac{\cos (6 \theta)}{L}\right. \\
& \left.+\frac{9}{64} \frac{\cos (7 \theta)}{L}+\frac{1}{8} \frac{\cos (8 \theta)}{L}+\frac{7}{64} \frac{\cos (9 \theta)}{L}\right]
\end{aligned}
$$




$$
\begin{aligned}
v(s, \theta)= & B N_{1 i} W_{1 i}+B N_{2 i} W_{2 i}+B N_{2 i}^{\prime} \varphi_{2 i}+B N_{3 i} W_{3 i}+B N_{3 i}^{\prime} \varphi_{3 i}+B N_{4 i} W_{4 i}+B N_{4 i}^{\prime} \varphi_{4 i}+B N_{5 i} W_{5 i} \\
& +B N_{1 j} W_{1 j}+B N_{2 j} W_{2 j}+B N_{2 j}^{\prime} \varphi_{2 j}+B N_{3 j} W_{3 j}+B N_{3 j}^{\prime} \varphi_{3 j}+B N_{4 j} W_{4 j}+B N_{4 j}^{\prime} \varphi_{4 j}+B N_{5 j} W_{5 j} \\
w(s, \theta)= & N_{1 i} W_{1 i}+N_{2 i} W_{2 i}+N_{2 i}^{\prime} \varphi_{2 i}+N_{3 i} W_{3 i}+N_{3 i}^{\prime} \varphi_{3 i}+N_{4 i} W_{4 i}+N_{4 i}^{\prime} \varphi_{4 i}+N_{5 i} W_{5 i}+N_{1 j} W_{1 j} \\
& +N_{2 j} W_{2 j}+N_{2 j}^{\prime} \varphi_{2 j}+N_{3 j} W_{3 j}+N_{3 j}^{\prime} \varphi_{3 j}+N_{4 j} W_{4 j}+N_{4 j}^{\prime} \varphi_{4 j}+N_{5 j} W_{5 j}
\end{aligned}
$$

118 All other shape functions are determined in the same form. This formulation presents a simple formula for

$$
\{v w\}^{\mathrm{T}}=[N] \times\{\delta\}
$$

As referred previously, the mechanical deformation model considers that the pipe undergoes a semi-membrane strain field by Eqs. (4-5). The typical use of the principle of virtual work leads to the system of algebraic equations. Having solved the system of algebraic equations, the displacement field is obtained for all the nodes of the finite tubular ring element.

\section{Deformation models on pipes design}

One step of design is resistance to loads imposed on the pipe during transportation and installation. The most common load is the diametral F-load, see Fig. 2. This load appears when pipes are stacked or when soil is compacted on the sides or on top of the pipe (Watkins and Anderson, 2000). If yield strength of the pipe material is exceeded due the F-load, either the pipe wall will crack or the cross section of the pipe will permanently deform. Either of these deformations, a crack is a deformation but unacceptable. The yield strength may possible be a performance limit even though the ring does not collapse.

The limit of the F-load for some manufactures pipes is based on the maximum allowable ring deflection based on Eq. (15). For the design process of the pipe transportation/installation loads, the maximum allowable F-load for plain pipes is calculated by the expression 16 (Watkins and Anderson, 2000).

$$
d^{\prime}=d / D \text { and } F=\left(\pi \sigma_{c} D / 3\right)(t / D)^{2}
$$

The ring defection for mechanical analysis of thin-walls rings with symmetrical loads is given by:

$$
d^{\prime}=0.0186\left(F D^{2} / E I\right)
$$

where $d^{\prime}$ represents the ring deflection, $d$ the decrease in diameter, $D$ the mean diameter of the pipe, $t$ the wall thickness for plain pipe, $\sigma_{c}$ is the yield strength and $E I$ the wall stiffness per unit of length of the pipe.

\section{Case studied 1: pinched cylinder}

The pinched cylinder problem tests the ability of simulation to model complex bending and membrane 148 states. In the pinched cylinder, the shear-locking phenomenon is more detrimental to the solution accuracy than membrane-locking. A severe problem for most finite elements is the locking which can appear as shear or membrane locking, as reported by Andelfinger and Ramm (1992). Transverse shear locking can occur in
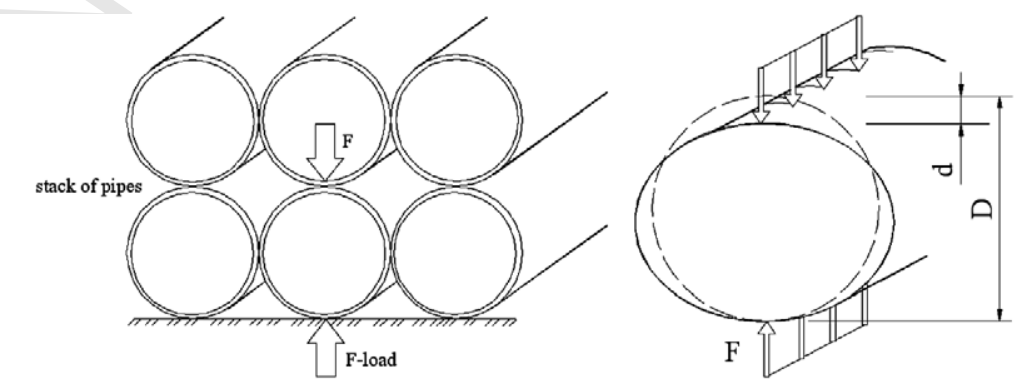

Fig. 2. Cylinder submitted to axisymmetric compression load: common transportation/installation on pipes. 

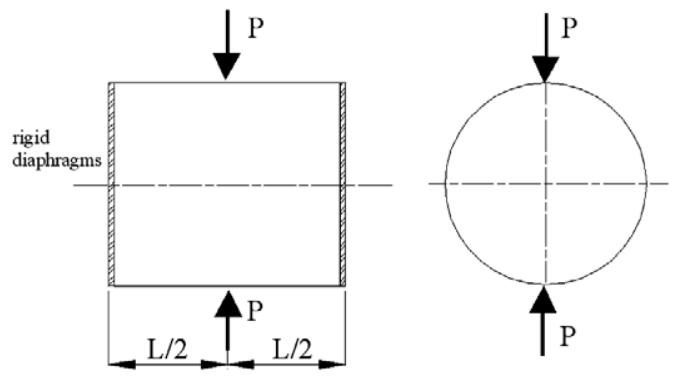

Fig. 3. Geometry of a pinched cylinder loaded.

149 shear deformable beam, plate and shell elements or solid elements if these are applied to the analysis of thinwalled structures. Transverse shear locking is one of the most important locking effects because it can essentially exclude an analysis with a reasonable amount of numerical effort in practical applications. This effect is significant only if there is a certain in-plane bending deformation of the structure. Shear locking occurs where the shear stiffness is a lot higher than the bending stiffness. Membrane locking can occur in thin shells or curved elements where the membrane stiffness is a lot higher than the bending stiffness. Researchers use the pinched cylinder problem as a benchmark test to assess the performance of curved beam or shells elements. The problem here presented, concerns a pinched cylinder with rigid end diaphragms, subjected to diametrical load as shown in Fig. 3, proposed by MacNeal and Harder (1985) and studied by a several authors. The geometric configuration of the cylinder is the length ( $L=600 \mathrm{in}$.), the radius ( $r=300 \mathrm{in}$.) and wall thickness ( $t=3$ in.). The elastic modulus was taken $\left(E=3 \times 10^{6} \mathrm{psi}\right)$ and Poisson's ratio $(v=0.3)$. Only a half of the cylinder is modelled due the geometry and load symmetry. The point load applied is equal $(P=1 / 2 \mathrm{lb})$. The reference displacement, as reported by Dvorkin and Bathe (1984), Kouhia and Stenberg (2000) and the exact deflection at the load point is equal $(0.18248 \mathrm{E}-4$ in. $)$, using $w_{\text {exact }}=164.24(P / E t)$. This is known as a benchmark result where there is a bending dominant behaviour in the thin shell limit. Thus transverse shear and membrane locking occurs as the shell becomes thinner. Fig. 4 represents the vertical displacement along length of cylinder obtained with our formulation with two different meshes and shell element from Cos$\operatorname{mos} / \mathbf{M}^{\circledR}$.

167 Table 1 shows the normalized vertical displacement under the load of the pinched cylinder modelled using 168 our proposed element and other references. Fig. 5 shows the vertical displacement using Cosmos/M ${ }^{\circledR}$. The solution obtained by the ring element does not exceed the analytical solution because it does not include transverse shear deformation. Thus, the numerical results of the present element are also compared with another solution, using a shell element from Cosmos $/ \mathrm{M}^{\circledR}$ which allows transverse shear deformation. The result of the displacement using ring element performs well even in the coarse mesh configurations. The small slenderness

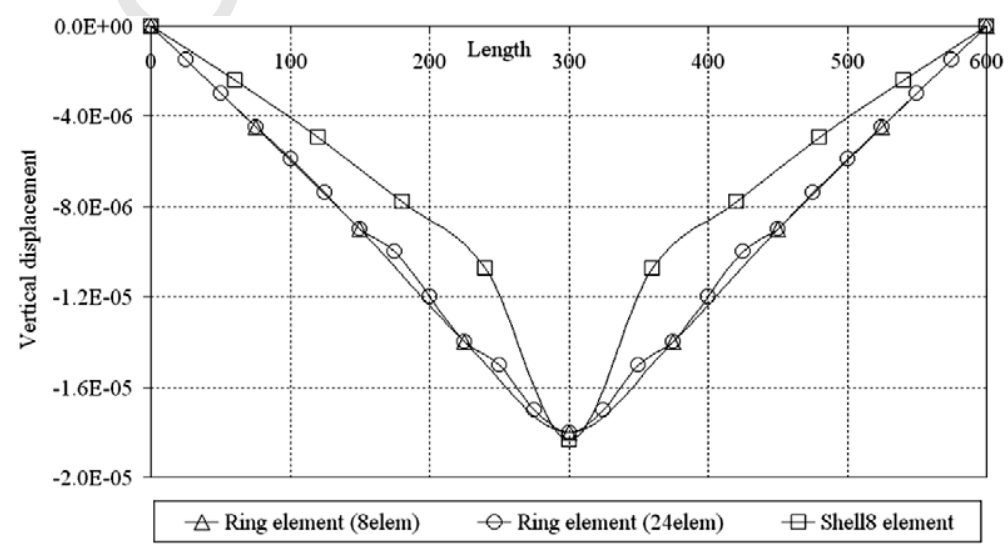

Fig. 4. Displacement with our formulation and Cosmos/ $\mathbf{M}^{\circledR}$. 
Table 1

Normalized displacement under the load

\begin{tabular}{ll}
\hline Meshes & $w_{\text {FEM }} / w_{\text {exact }}$ \\
\hline $5 \times 5^{\mathrm{a}}$ & 0.5100 \\
$10 \times 10^{\mathrm{a}}$ & 0.8300 \\
$20 \times 20^{\mathrm{a}}$ & 0.9600 \\
$4 \times 4^{\mathrm{b}}$ & 1.1769 \\
$8 \times 8^{\mathrm{b}}$ & 0.9912 \\
$16 \times 16^{\mathrm{b}}$ & 1.0059 \\
Ring element $(8$ or 24 elements $)$ & 0.9864 \\
Shell8 element Cosmos $/ \mathrm{M}^{\circledR}(400$ elements $)$ & 1.0060
\end{tabular}

${ }^{a}$ Dvorkin and Bathe (1984).

b Kouhia and Stenberg (2000).
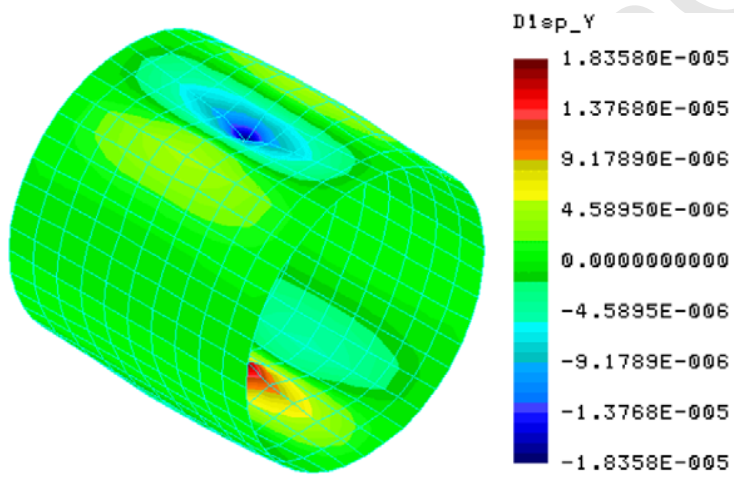

Fig. 5. Vertical displacement using $\operatorname{Cosmos} / \mathrm{M}^{\circledR}$.

173 ratio $(t / r=1 / 100)$ of the cylinder is chosen to demonstrate the capability of our ring element to overcome 174 shear and membrane-locking phenomena.

\section{6. Case studied 2: ring deformation}

176 The studied case of a ring deformation is shown in Fig. 6, representing a tubular steel ring structure sub177 mitted to a F-load along the length $(L=170 \mathrm{~mm})$ and free ends. The tubular section presents an external 178 diameter $(\mathrm{DE}=165 \mathrm{~mm})$ and an internal diameter $(\mathrm{DI}=155 \mathrm{~mm})$. The elasticity modulus is $(E=2.1 \mathrm{GPa})$ 179 and the Poisson coefficient is equal to $(v=0.3)$. The theoretical value of the decrease diameter $(d)$ on the 180 top section and the lateral displacement at middle of section $(u)$ can be obtained using the Castiglino's 181 theorem:
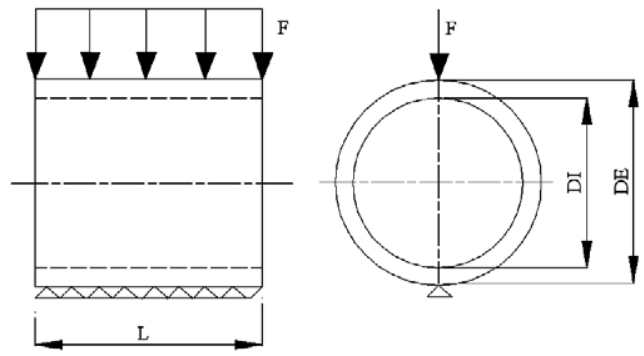

Fig. 6. Parameters for ring structure. 


$$
\begin{aligned}
& d=-\left[\frac{\operatorname{Pr}^{3}\left(\pi^{2}-8\right)}{8 \pi E I}+\frac{\operatorname{Pr} \pi}{8 G A m}+\frac{\operatorname{Pr} \pi}{8 E A}\right] \\
& u=\frac{\operatorname{Pr}^{3}(4-\pi)}{4 \pi E I}+\frac{\operatorname{Pr}}{4 G A m}-\frac{\operatorname{Pr}}{4 E A}
\end{aligned}
$$

185 In this case: $I=L t^{3} / 12, A=t L, G=E / 2(1+v)$ and $m=9 / 10$ is the correction factor. The transversal displacement at the top and the lateral displacement will be analysed using the experimental method, analytical solutions and numerical results obtain with the developed finite ring element. The experimental setup used is represented in Fig. 7. The loading system is implemented by a hydraulic jack and load cell, and both displacements are obtained with a data acquisition system using two LVDT's.

Fig. 8 represents the decrease displacement in diameter function of the F-Load, obtained with five experimental tests. The elastic part of the experimental results may be compared with the results of Eq. (15) or (18) and numerical results from the finite ring element. Fig. 9 represents the lateral displacement measured for the same five experimental tests. The elastic part of the experimental results may be compared with the results provided by Eq. (19) and the numerical results obtained from the finite ring element.

The experimental tests present satisfactory results when compared with the numerical and analytical solution. Increasing loading test will gradually increase scattered data results. Numerical results approach the theoretical curve, while the experimental results are positioned below this curve. The experimental results present a non-linear trend for higher load values. Linear data can be useful to determine pipe stiffness, defined as resistance to ring deflection.

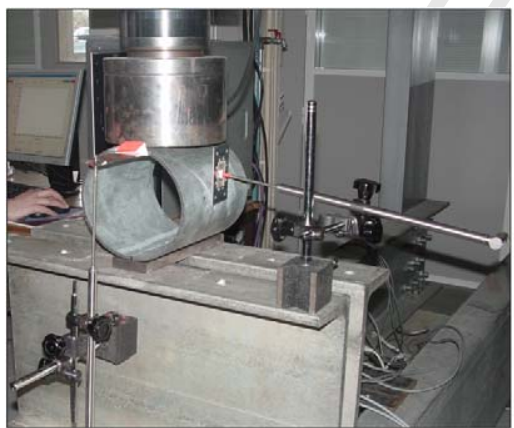

LVDT's (max.linearity deviation $0.12 \%$ ).

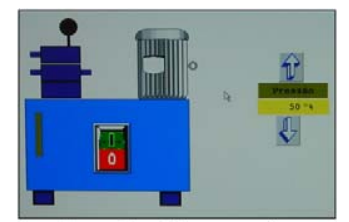

Load cell control.

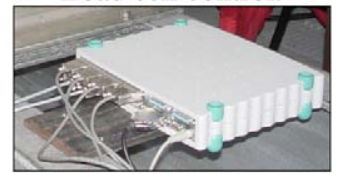

Data acquisition.

Fig. 7. Experimental assembly for ring structure.

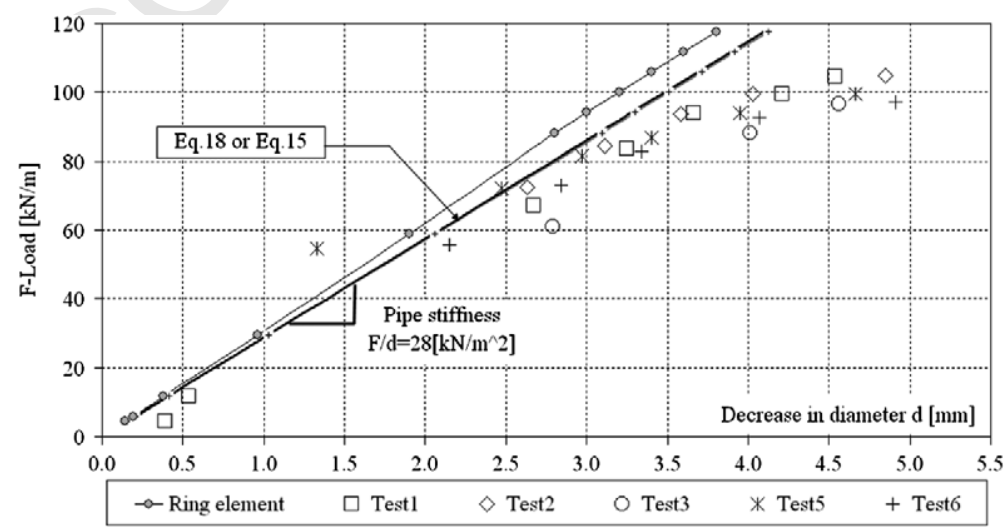

Fig. 8. Decrease displacement in diameter $d$. 


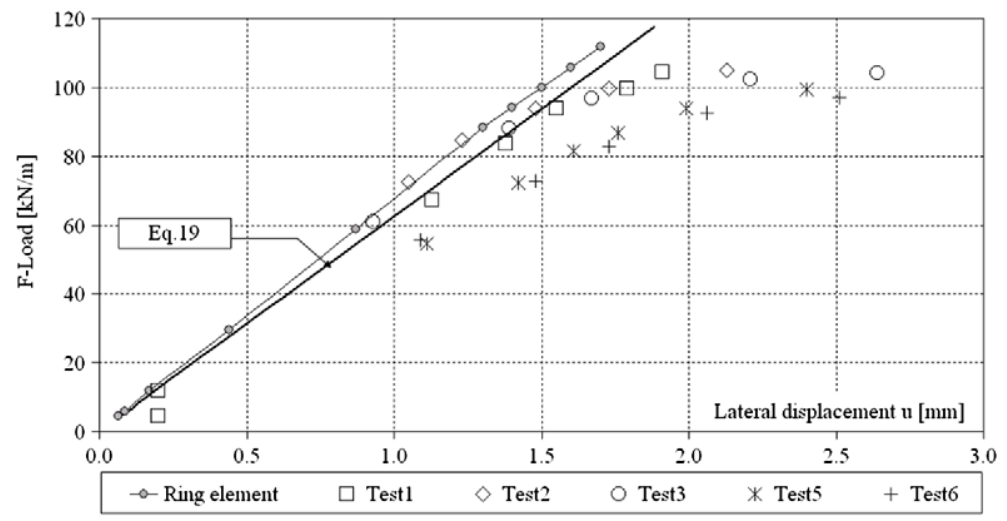

Fig. 9. Lateral displacement $u$.

\section{Conclusion}

A computer program based on the linear theoretical formulation described was developed for the analysis and determination of the transverse displacement field along ring section of tubular pipes. To validate the element accuracy, numerical results were presented with a good agreement with other authors, analytical solutions and numerical analysis using $\operatorname{Cosmos} / \mathrm{M}^{\circledR}$. The presented solution has performed well even with coarse element meshes, and appears as a simple and easy-to-handle alternative to the use of shell or beam finite elements due the capability and effectiveness of this element. The presented formulation using a multi-nodal element under symmetric conditions makes possible to determine a displacement field under shell surface for in-plane bending problems. A pre-processor for generate one-dimensional meshes with two sections $(i$ and $j)$ per element is only necessary. The Cosmos $/ \mathrm{M}^{\circledR}$ shell element presents eight nodes per element, and a more refined three-dimensional mesh is necessary. The ring element exhibits excellent behaviour for all configurations in linear bending conditions like all shell elements, when using a small slenderness ratio between thick-

\section{References}

Ahmad, S., Irons, B.M., Zienkiewicz, O.C., 1970. Analysis of thick and thin shell structures by curved finite elements. Int. J. Numer. Meth. Eng. 2, 419-451.

Andelfinger, U., Ramm, E., 1992. An Assessment of hybrid-mixed four-node shell elements. In: Rammerstorfer, F.G. (Ed.), Nonlinear Analysis of Shells by Finite Elements. Springer-Verlag, New York, pp. 31-45.

Bathe, K.J., Almeida, C.A., 1982. A simple and effective pipe elbow element - Pressure stiffening effects. J. Appl. Mech. 49, 914-916.

Cheng, D.H., Thailer, M.J., 1970. On the bending of curved circular tubes. ASME J. Eng. Ind. B 92 (1), 62-66, B.

Dvorkin, E., Bathe, K.J., 1984. A continuum mechanics based four-node shell element for general non-linear analysis. Eng. Comput 1, 7788.

Fonseca, E.M.M., Melo, F.J.M.Q., Oliveira, C.A.M., 2002. Determination of flexibility factors on curved pipes with end restraints using a semi-analytic formulation. Int. J. Pressure Vessels Piping 79 (12), 829-840.

Fonseca, E.M.M., Melo, F.J.M.Q., Oliveira, C.A.M., 2005. The thermal and mechanical behaviour of structural steel piping systems. Int. J. Pressure Vessels Piping 82 (2), 145-153.

Gobetti, A., Nascimbene, R., 2001. Elasto-plastic, nonlinear analysis of a locking-free shear/flexible curved beam element. In: ECCMEuropean Conference on Computational Mechanics, Poland.

Kim, K.D., Liu, G.Z., Han, S.C., 2004. A resultant 8-node solid-shell element for geometrically nonlinear analysis. Comp. Mech. 35 (5), 315-331.

Kouhia, R., Stenberg, R., 2000. A simple linear nonconforming shell element. In: IASS-IACM 2000, Fourth International Colloquium on Computational of Shell Spatial Structures, Greece.

MacNeal, R.H., 1998. Perspective on finite elements for shell analysis. J. Finite Elem. Anal. Des. 30, $175-186$.

MacNeal, R.H., Harder, R.L., 1985. A proposed standard set of problems to test finite element accuracy. J. Finite Elem. Anal. Des. 1, 320.

Melo, F.J.M.Q., Castro, P.M.S.T., 1992. A reduced integration Mindlin beam element for linear elastic stress analysis of curved pipes under generalized in-plane loading. Comp. Struct. 43 (4), 787-794. 
Oñate, Eugenio., 1992. Cálculo de Estructuras por el Método de Elementos Finitos. Structure Calculation using the Finite Element Method. CIMNE Edition, The Polythecnic University of Calaunia, chapts 10, a2 and 14 (in spanish).

Thomson, G., 1980. The influence of end constraints on pipe bends. Ph.D. thesis, University of Strathclyde, Scotland, UK.

Ugural, A.C., 1981. Stresses in Plates and Shells. McGraw-Hill Inc.

Vigness, I., 1943. Elastic properties of curved tubes. ASME 65, 105-120.

von Kárman, Th., 1911. Über die formanderung dunnwaindiger rohre insbesondere federnder ausgleichrohre. Zeits V.D.I. Band 55, 8891895.

Watkins, R.K., Anderson, L.R., 2000. Structural Mechanics of Buried Pipes. CRC Press, New York.

Wilczek, E., 1984. Statische berechnung eines rohrkrümmers mit realen randbedingungen. Ph.D. thesis, Institut für Leichtbau, Aachen. 